\title{
Experimental research on a hot air generator working on the TLUD principle
}

\author{
Ioan Pavel ${ }^{1,}$, Radu-Iulian Rădoi ${ }^{1}$, Gabriela Matache $^{1}$, Ana-Maria Carla Popescu ${ }^{1}$, and \\ Ioan $\mathrm{Caba}^{2}$ \\ ${ }^{1}$ Hydraulics and Pneumatics Research Institute INOE 2000-IHP, 040558, Cutitul de Argint 14, \\ Bucharest, Romania \\ ${ }^{2}$ National Institute of Research - Development for Machines and Installations Designed to Agriculture \\ and Food Industry - INMA, 71592, 6 Ion Ionescu de la Brad, Blv, Bucharest, Romania
}

\begin{abstract}
The current paper presents the processes of data acquisition and air temperature monitoring in the key points of a hot air generator working on the TLUD principle, which uses temperature sensors, a data acquisition board and an application developed in LabView that numerically and graphically displays temperature variation during burning. The results of the monitoring were obtained on an experimental model, developed by research scientists at INOE 2000-IHP. The data acquired is then used to establish the algorithm for opening the air supply valves for gasification and combustion air, in order to optimize combustion by maintaining the optimum temperature at the funnel.
\end{abstract}

\section{Introduction}

The use of renewable energies is a characteristic of contemporary society, which is facing the decrease of classical resources, the rise in fuel prices and the pollution generated by their burning. However, the use of renewable resources to provide energy needs is still far from its full potential; this is also because of the lack of efficient conversion tools able to exploit various resources in mono-source or combined energy systems [1].

In the process of gasification, the inputs are biomass and air, while the outputs are fuel gas, biochar and ash, with a negative balance of carbon emissions due to carbon sequestration by biochar. The TLUD gasification principle (Figure 1) occurs when the biomass layer is introduced into a reactor and rests on a grid through which the air flow for gasification circulates from bottom to top.

Priming gasification process is done by igniting the top layer of the biomass in the reactor. The combustion front descends continuously by consuming the biomass in the reactor. Due to the heat radiated from the oxidation front the biomass is heated, dried, and then it enters a fast pyrolysis process that releases volatiles and there remains unconverted carbon.

\footnotetext{
* Corresponding author: pavel.ihp@,fluidas.ro
} 
By the time the combustion front reaches the grill all the volatiles in the biomass were gasified and some of the fixed carbon was reduced; about $10-20 \%$ of the initial mass remain on the grill, in the form of sterile "green charcoal", called biochar.

The proportion of biochar, in which most of the ash from biomass is incorporated, depends both on the carbon stored in the biomass and on the temperature maintained in the carbon reduction reaction; a high temperature ensures the reduction of more carbon. If the supply with gasification air continues, we switch to an updraft gasification process in which a layer of incandescent charcoal is kept on the grill; of this, there results mainly $\mathrm{CO}$ and little $\mathrm{CO} 2$, which passing through the hot charcoal layer enters the reduction reaction $\mathrm{C}+\mathrm{CO}_{2} \Rightarrow 2 \mathrm{CO}$. This second phase is called charcoal gasification.

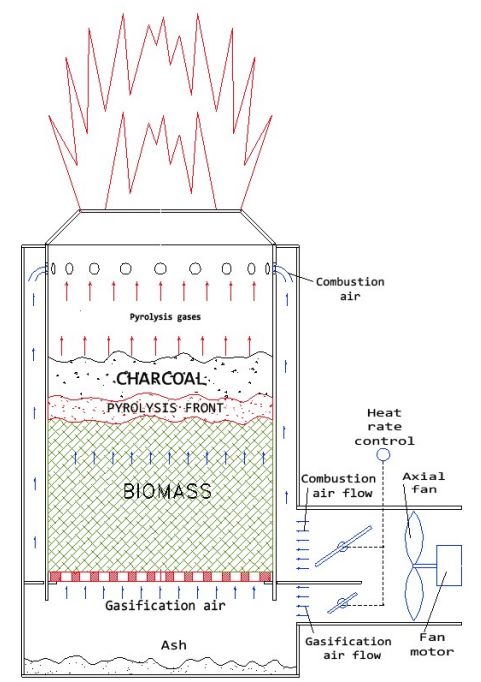

Fig. 1. The TLUD gasification principle [2].

Compared to direct combustion or gasification processes of wood and pellets, the TLUD gasification process is characterized by very low values of the superficial gas velocity through the oxidation front, resulting in a very low content of atmospheric particulate matter $(\mathrm{PM}), \mathrm{PM}<5 \mathrm{mg} / \mathrm{MJ}$, well below the required standard in the EU since 2015 for biomass combustion processes, which is $25 \mathrm{mg} / \mathrm{MJ}$.

The calculation algorithm of thermal power is [1]:

Reactor diameter:

$$
D_{r}=0.35 m
$$

Biomass layer height (batch height):

$$
H_{r b m}=0.6 m
$$

Reactor section:

$$
S_{r}=\frac{\pi \cdot D_{r}^{2}}{4}=\frac{\pi \cdot 0.35^{2}}{4}=\frac{0.3847}{4}=0.0961 \mathrm{~m}^{2}
$$

Biomass volume in the reactor: 


$$
V_{r b m}=H_{r b m} \cdot S_{r}=0.6 \cdot 0.0961=0.0577 \mathrm{~m}^{3}
$$

Biomass layer density: $600 \mathrm{~kg} / \mathrm{m}^{3}$ (pellets).

Initial mass in the reactor:

$$
M_{b m 0}=600 \cdot 0.05766=34.6 \mathrm{~kg}
$$

The specific hourly consumption of gasified biomass is $85 \mathrm{~kg} / \mathrm{m}^{2} \cdot \mathrm{h}$; therefore, for the surface of the reactor we will have a specific consumption of:

$$
C_{h b m g}=85 \cdot 0.0961=8.17 \mathrm{~kg} / \mathrm{h}
$$

The running time will be:

$$
t_{g}=\frac{34.6}{8.17}=4.23 h
$$

Energy from gasified biomass:

$$
E_{g b m}=M_{b m 0} \cdot P_{C b m}=34.6 \cdot 17=588.2 M J
$$

Thermal power of hot gases:

$$
P_{g}=\frac{E_{g b m}}{t_{g} \cdot 3.6} \cdot \eta_{g T L U D}=\frac{588.2 \cdot 0.93}{4.23 \cdot 3.6}=35.922 \mathrm{kWth}
$$

and burner thermal power, which takes into account the efficiency of combustion (comb) of the combustible gas $\left(\eta_{\text {comb }}=0.95\right)$ and the insulation (insul) efficiency (losses outward $\eta_{\text {insul }}=0.96$ ), will be:

$$
P_{\text {comb }}=P_{g} \cdot \eta_{\text {comb }} \cdot \eta_{\text {insul }}=35.922 \cdot 0.95 \cdot 0.96=32.76 \mathrm{kWth}
$$

The gasification process is done with a reduced intensity with a specific hourly consumption of biomass of $80-150 \mathrm{~kg} / \mathrm{m}^{2} \mathrm{~h}$, which leads to reduced specific powers of 250 - $350 \mathrm{~kW} / \mathrm{m}^{2}$ of the reactor. The slow process maintains the superficial speed of the generator gas produced at very low values, $\mathrm{v}_{\text {sup }} \leq 0.06 \mathrm{~m} / \mathrm{s}$, which ensures reduced traction of free ash, and also at concentrations of $\mathrm{PM}_{2.5}$ of maximum $5 \mathrm{mg} / \mathrm{MJ}_{\mathrm{bm}}$ when leaving the burner; this value is at least five times lower than current standards required for solid fuel heat generators. [3, 4, 5, 6, 7]

The stages of the gasification process take place simultaneously in different areas of the reactor. These stages are: drying, pyrolysis, oxidation and reduction.

Drying is necessary because the moisture content of the biomass is variable, ranging from 5 to $55 \%$. At temperatures above $100^{\circ} \mathrm{C}$, the water is removed and turned into steam. During the drying process, the biomass does not suffer any decomposition.

Pyrolysis takes place in the temperature range of $150-700^{\circ} \mathrm{C}$, and it consists of thermal decomposition of biomass in the absence of oxygen.

Oxidation takes place by the aid of the air introduced into the oxidation zone. The air contains, together with oxygen, water vapor, inert gases, nitrogen and argon that do not react with the biomass components. Oxidation takes place at $700-2000^{\circ} \mathrm{C}$. 
Reduction occurs in the reduction zone of the reactor. Here several chemical reactions take place at a temperature of $800-1000^{\circ} \mathrm{C}$ and in the absence of oxygen.

The generator gas is a mixture of combustible and non-combustible gases. The combustible gases are: carbon monoxide $(15-30 \%)$, hydrogen $(10-20 \%)$, methane $(2$ $4 \%)$. The non-combustible gases are: nitrogen (45-60\%), water vapors $(6-8 \%)$, carbon dioxide $(5-15 \%)$ [8].

\section{The objective of testing}

Until now, the TLUD gasification principle has been applied mostly to small and very small food preparation equipment. The prototype of hot air generator on the TLUD principle under research is a $32-\mathrm{kW}$ generator, so it is necessary to test the principle for such a size, in different operating modes.

Testing the prototype of hot air generator on the TLUD principle aims to acquire temperature data from key points, in two variants, that is with and without getting biochar.

Temperature monitoring is done for the following areas (Figure 2):

- Environment

- Combustion air inlet and gasification air inlet

- Hot air outlet

- Funnel.

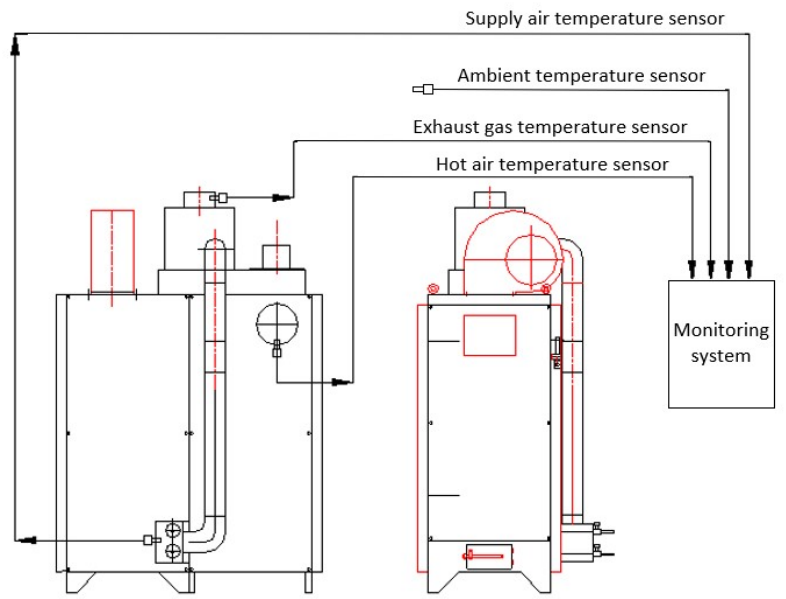

Fig. 2. Positioning of temperature sensors.

The acquired data is then used to establish the opening algorithm for gasification air supply valves and combustion air supply valves, in order to optimize combustion by keeping the optimum temperature at the funnel.

The generator consists of a reactor (7) in which the gasification of fuel from biomass (in this case pellets) occurs, a burner (6) and a heat exchanger (1). It is equipped with a fan for forced draft along the funnel (3) and another one for the circulation of hot air through the heat exchanger (5). The gasification air is controlled at the inlet (9), and the combustion air - at the inlet (8). The hot air leaves the equipment through the outlet (2), and the smoke through the funnel (4). 

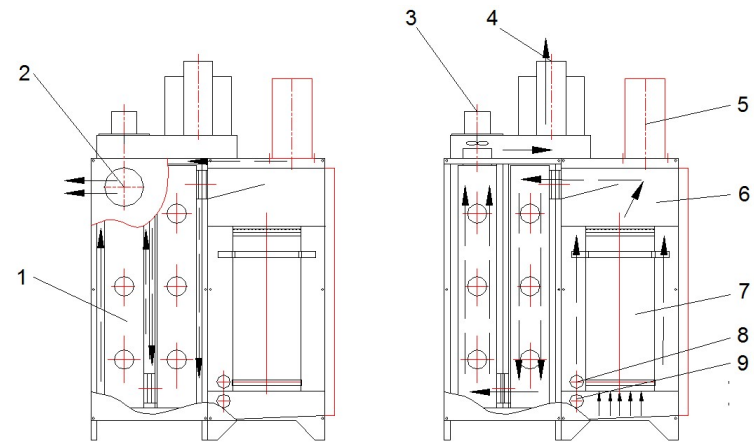

Fig. 3. Hot air generator working on the TLUD principle.

The correct operation of the hot air generator implies keeping a temperature of approximately $200^{\circ} \mathrm{C}$ at the funnel to avoid condensation and tar deposition. It is important to adjust the air at the outlets 8 and 9 in order to meet this requirement. If the temperature is lower, tar is deposited, and if it is higher, unnecessary energy is lost to the atmosphere.

Opening of the valves for combustion air and gasification air intake has been made by measuring the opening slit in such a way that the ratio of air set to the valves is Qa / Qg = 2.62, where $\mathrm{Qa}$ is the combustion air flow rate, and $\mathrm{Qg}$ is the gasification air flow rate. Specialized literature [9], [10] indicates that the flow rate ratio must be in the range $2.44 \ldots$ 3.3 to get the best results. From the same sources it is known that the superficial velocities of the synthesis gas are from $0.03 \mathrm{~m} / \mathrm{s}$ to $0.04 \mathrm{~m} / \mathrm{s}(\mathrm{PM}<5 \mathrm{mg} / \mathrm{MJ})$, far below the critical level of $0.1 \mathrm{~m} / \mathrm{s}$ where the specific $\mathrm{PM}_{2.5}$ concentration increases above $2.4 \mathrm{mg} / \mathrm{MJ}$. Upon leaving the reactor, the flue gas has a flow rate of $0.007 \mathrm{~m}^{3} / \mathrm{s}$ and an average temperature of $695^{\circ} \mathrm{C}[11]$.

Testing consists of data acquisition and monitoring of air temperatures at key points for the two operating modes: minimum and maximum power. Power control is done by controlling the opening of the combustion air and gasification air intake valves.

\section{Data acquisition}

Recording the temperature evolution at various points of the hot air generator is done by means of Pt1000 temperature probes. They are connected to a data acquisition board via signal amplifiers of $4 \ldots 20 \mathrm{~mA}$ output, and voltage conversion for the analog input of the data acquisition board is achieved by means of resistors (Figure 4).

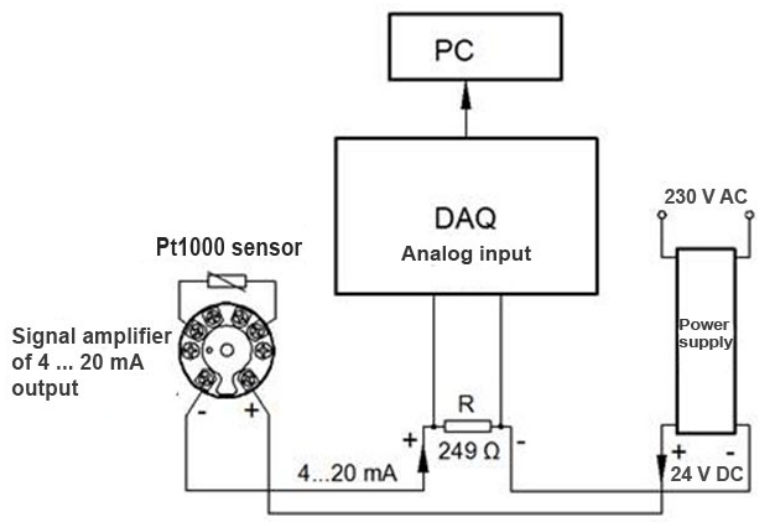

Fig. 4. Connection diagram. 
A $24 \mathrm{~V} \mathrm{DC}$ power supply was used to power the acquisition board and the amplifier [12]. The technical features of the signal amplifier code 2000.35.010 transducer RTD4 ...20 $\mathrm{mA}$ are according to the technical sheet [13].

The signal from the temperature sensor is amplified in a $4 \ldots 20 \mathrm{~mA}$ output signal. The data acquisition board (DAQ M Series NI USB-621x [14]) allows inputs of 0-10 V, and the amplified signal of 4-20 mA was converted into voltage by means of a resistor of $249 \Omega$, as calculated:

$$
\begin{gathered}
20 m A=0.02 A \\
0.02 A \times 249 \Omega=5 V
\end{gathered}
$$

(the middle of the permissible voltage range of the acquisition board, which is $0 \ldots 10 \mathrm{~V}$ )

An application in LabView (Figure 5) was created to display and record data. The application displays the temperature variation during the burning process numerically and graphically. When the recording stops, the application allows the user to store the temperature versus time values in a database. These data may be further processed.

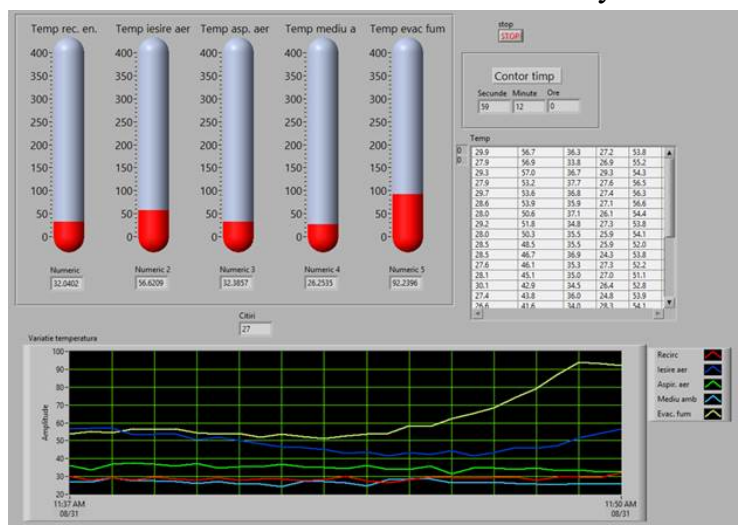

Fig. 5. Graphical user interface of the data acquisition software application. 


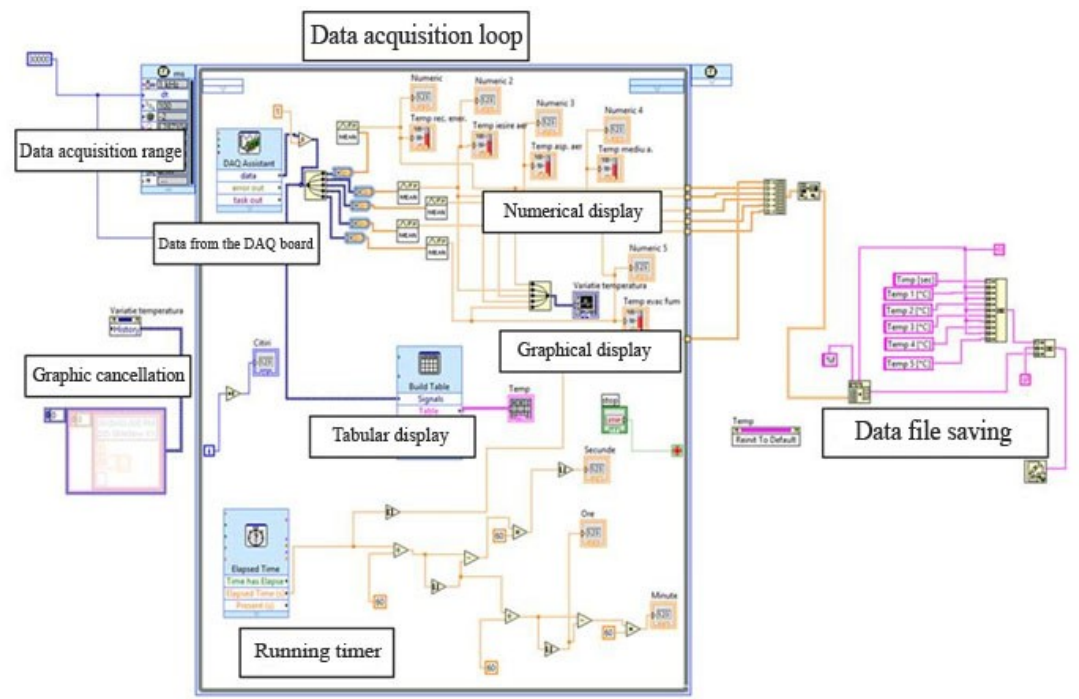

Fig. 6. Functional diagram of the application developed in LabView.

The application developed in LabView comprises the following functional blocks (Figure 6): a data acquisition loop with the functionality to set the range of data acquisition, an input block for data from the acquisition board in which there are also performed temperature - output signal scaling, numerical display, graphical display, running timer (seconds, minutes, hours), data recording in text file, cancellation of the graphic (when starting a new test), and a block for data display in tabular format.

\section{Actual testing with data acquisition}

The contents of a $15 \mathrm{~kg}$ bag of pellets were introduced into the reactor. Initiation of the combustion process was done at the top of the material in the reactor, by using firelighters, and from ignition to steady gasification operation mode (Figure 7) it took about 12 minutes according to the data acquisition.

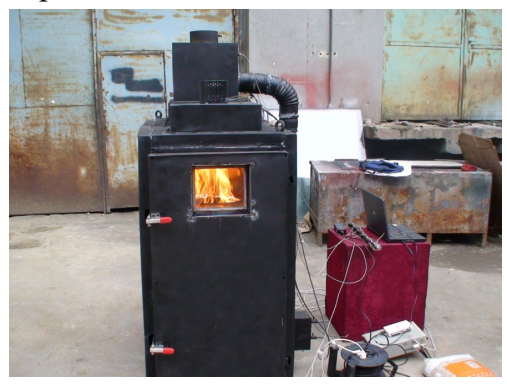

Fig. 7. The steady gasification operation mode

\subsection{Test no. 1- Data acquisition in the variant of gasification with biochar production under minimal operating conditions}


After entering the gasification operation mode, the flame has moved from the combustion material in the reactor to the burner (Figure 8.a). The gas produced in the reactor mixed with the combustion air, and a flame similar to a stove one was generated. Successive adjustment of the valves for gasification air and combustion air intake has been done (Figure 8.b) keeping the ratio Qa / Qg=2.62, and it was found that the power of the hot air generator was adjustable. When opening the valves one can see on the graphical interface, through the acquisition system (Figure 8.c), that the flame increases almost instantaneously. The correct operation of the TLUD (Top-Lit UpDraft) gasification principle has thus been demonstrated.

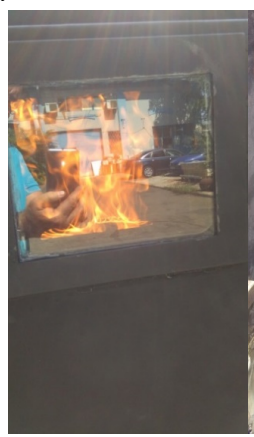

(a)

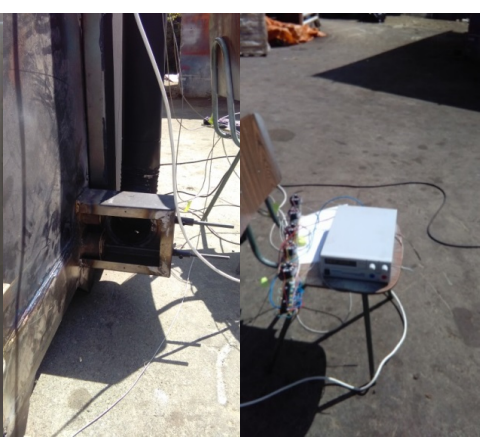

(c)

Fig. 8. (a) Adjustment of air intake valves, (b) Steady gasification operation mode, (c) Data acquisition system.

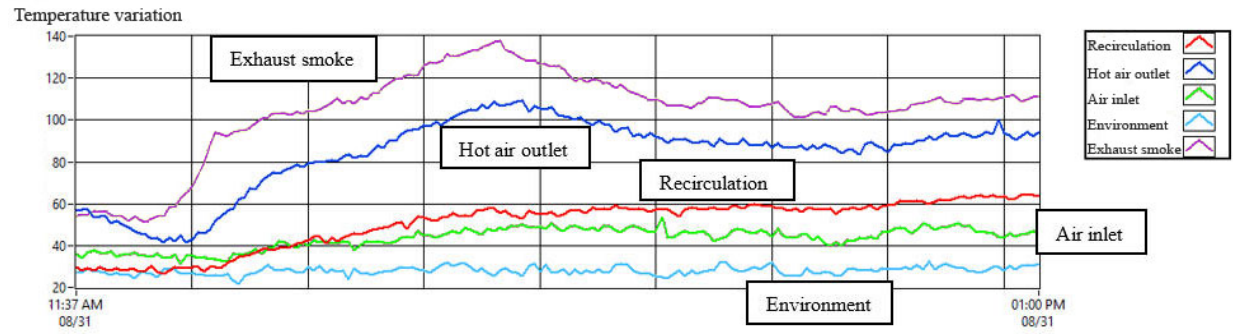

The data acquisition resulted in the following graph and related database.

Fig. 9. The data acquisition graph for the test with obtaining of biochar.

A good adjustment of the power of the hot air generator has been obtained by setting at minimum the combustion air and the gasification air. The temperature of the heating air at the output of the generator, for the case of minimum operating conditions, registered values of approx. $110^{\circ} \mathrm{C}$, and on the funnel, a temperature of approx. $140^{\circ} \mathrm{C}$ was obtained. The air intake valves were set to approximately 1-2 mm opening.

The combustion process (Figure 9) took 83 minutes for a $15 \mathrm{~kg}$ bag of pellets under minimum power operating conditions (the intake valves - almost closed), and it was interrupted when the flame became blue (a sign that gasification of the material was over and burning of the biochar was about to start). Thus, about $25 \%$ of the volume of the material initially introduced for combustion was obtained as biochar. 
The biochar obtained can be seen in Figure 10 .

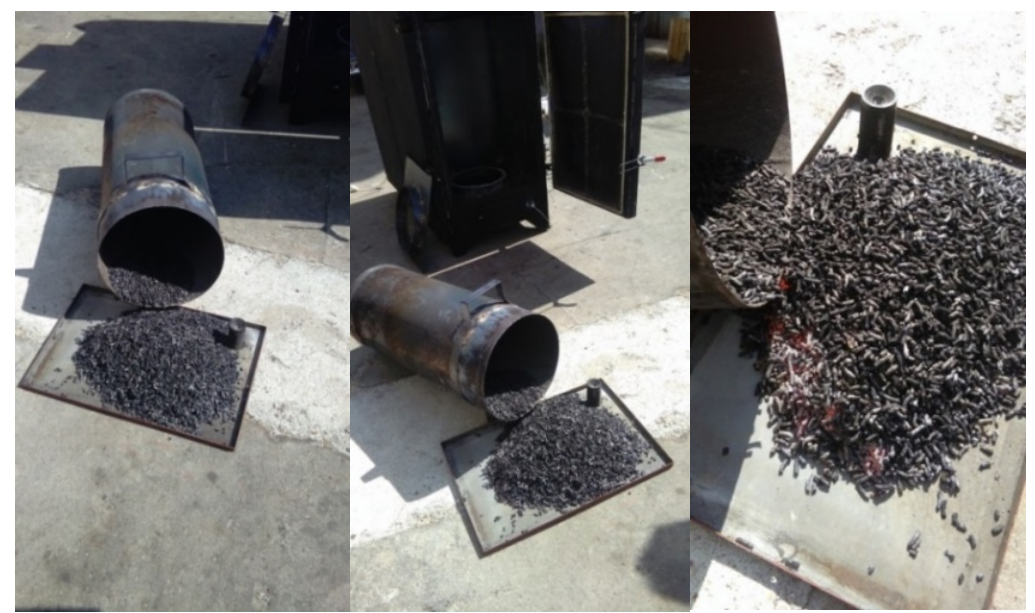

Fig. 10. Biochar obtained following gasification.

\subsection{Test no. 2 - Variant of gasification with burning of the biochar}

The contents of a $15 \mathrm{~kg}$ bag of pellets were introduced into the reactor. Initiation of the combustion process was done by using firelighters, and from ignition to steady gasification operation mode) it took about 12 minutes according to the data acquisition.

Under maximum power test conditions, that is when combustion air and gasification air intake valves open to more than $10 \mathrm{~mm}$, a heating air temperature of approx. $200^{\circ} \mathrm{C}$ was obtained, and a temperature of approx. $250^{\circ} \mathrm{C}$ was obtained on the funnel, according to the data acquisition graph (Figure 11).

In Test no. 2 the combustion process has not been interrupted any more to obtain biochar (when the flame became blue), and thus an operating time approximately equal to that of Test no. 1 was obtained, this time at maximum power, and the amount of energy obtained was higher by about $25 \%$ compared to the variant when the reactor was shutdown in order to produce biochar. The final result of the total combustion (including the biochar) was a very small amount of ash of about $50 \mathrm{~g}$ resulting from the combustion of a $15 \mathrm{~kg}$ bag of pellets.

When the pyrolytic front was close to the bottom of the reactor and the thickness of the biochar layer decreased, while maintaining the gasification air flow and the minimum resistance created by the material, the combustion process accelerated for a short time (until all the material including the biochar was consumed by combustion), and after that, although the combustion process became less intense, the generator still provided hot air for about 20 minutes due to thermal inertia.

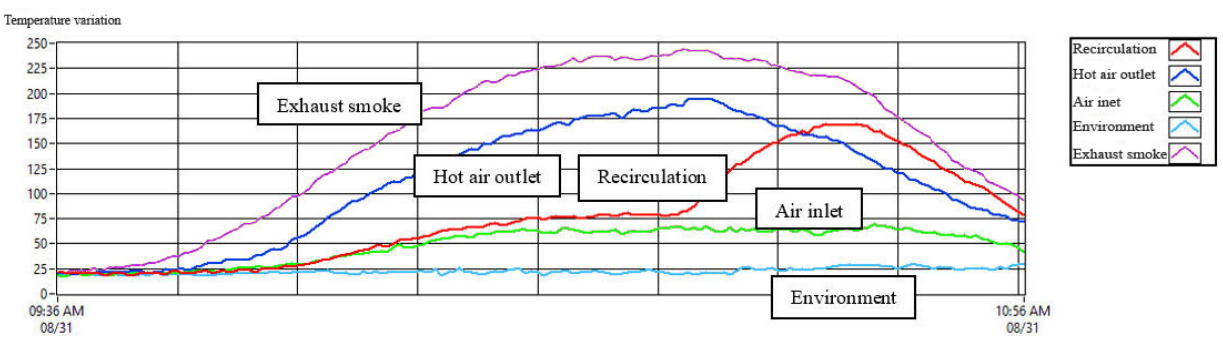

Fig. 11. The data acquisition graph for the test with burning of the biochar. 
During the tests, emission of pollutants was measured and no emissions of smoke or unburnt gases were found beyond legal limit.

\section{Conclusions}

The experimental model of $32 \mathrm{~kW}$ - hot air generator has been tested in the variant of minimum operating power with biochar production and in the variant of maximum operating power with gasification of the biochar, demonstrating the correct functioning of the TLUD principle and adjustability of the power of the generator.

The acquired data can be used in determining an algorithm for automatic opening of combustion air and gasification air intake valves, thus achieving system automation and correct operation of the TLUD principle. Another version can be with fixed adjustment of the air intake valves, keeping the $\mathrm{Qa} / \mathrm{Qg}=2.62$ ratio, for an average value of the power of the generator, provided that the fan flow rate for the forced draft on the funnel and the flow rate for the circulation of hot air through the heat exchanger can be varied. In this case, electronic air flow control is required in order to keep the temperature at the funnel around $200^{\circ} \mathrm{C}$.

Based on this research and the acquired data a simulation model can be developed to optimize the automation of combustion air and gasification air supply, so that the advantages of the TLUD principle (carbon emission negative balance, or obtaining of biochar) get optimal.

This paper has been developed in INOE 2000-IHP, as part of a project co-financed by the European Union through the European Regional Development Fund, under Competitiveness Operational Programme 2014-2020, Priority Axis 1: Research, technological development and innovation (RD\&I) to support economic competitiveness and business development, Action 1.2.3 - Partnerships for knowledge transfer, project title: Eco-innovative technologies for recovery of biomass wastes, project acronym: ECOVALDES, SMIS code: 105693, Financial agreement no. 129/23.09.2016.

\section{References}

1. L. Dumitrescu, C. Cristescu, V. Barbu, M. Mateescu, Proc. of International Symposium ISB-INMA'TEH, pp. 987-992 (2018)

2. G. Matache, I. Pavel, A. Pantiru, M. Ciciu, Hidraulica, 2, pp. 70-75 (2016) http://www.hidraulica.fluidas.ro/2016/nr2/70-75.pdf

3. A. Belonio, Dual- reactor rice husk gasifier for 6-ton capacity recirculating-type paddy dryer (Iloilo City, Philippines: Central Philippine University) https://stoves.bioenergylists.org/stovesdoc/Belonio/paddygas/Dual_Reactor_RH_Gasif ier.pdf

4. H. Mukunda, S. Dasappa, P. J. Paul, N. K. S. Rajan, M. Yagnaraman, D. R. Kumar, M. Deogaonkar, Current Science 98, 5, pp. 627-638 (2010)

5. E. Murad, A. Culamet, G. Zamfiroiu, Proc. of International Symposium HERVEX 2011, pp. 37-43 (2011)

6. J. Porteiro, D. Patino, J. Collazo, E. Granada, J. Moran, J.L. Miguez, Fuel 89, 1, pp. 26-35 (2010)

7. S. Varunkunar, Packed bed gasification-combustion in biomass domestic stove and combustion systems (PhD Thesis, Bangalore, India: Indian Institute of Science, Department of Aerospace Engineering, 2012) 
8. G. Matache, I. Pavel, A. Pantiru, M. Ciciu, Proc. of 5th International Conference of Thermal Equipment, Renewable Energies and Rural Development TE-RE-RD, pp. 277282 (2016)

9. F. Sundblad, An improved cooking stove for the urban and peri-urban areas in Zambia (Master of Science Thesis in Industrial Design Engineering, Gothenburg, Sweden: Chalmers University of Technology, 2014)

10. D. Supramono, F. Inayati, Proceeding of the 13th International Conference on QIR (Quality in Research), pp. 393-398 (2013)

11. E. Maican, I.C. Duţu, G. Matache, C. Dumitrescu, I. Pavel, INMATEH, 53, 3, pp. 5-12 (2017) http://www.inmateh.eu/INMATEH_3_2017/53-01\%20Maican\%20E.pdf

12. http://www.farnell.com/datasheets/43587.pdf?.ga $=2.205171536 .1703443844 .1508838$ 846-1396001712.1508838846

13. http://www.adelaida.ro/convertor-rtd-to-4-20ma-2300.10.176-pixsys.html

14. https://www.ni.com/pdf/manuals/371931f.pdf 